\title{
Surfaces
}

\section{Valdine Clemens, The Return of the Repressed: Gothic Horror from The Castle of Otranto to Alien. State University of New York Press, USA, 1999. 274 pages}

\section{Éric Alloi}

Volume 9, 2001

URI : https://id.erudit.org/iderudit/1065071ar

DOI : https://doi.org/10.7202/1065071ar

Aller au sommaire du numéro

\section{Éditeur(s)}

Les Presses de l’Université de Montréal

ISSN

1188-2492 (imprimé)

1200-5320 (numérique)

Découvrir la revue

Citer ce compte rendu

Alloi, É. (2001). Compte rendu de [Valdine Clemens, The Return of the Repressed: Gothic Horror from The Castle of Otranto to Alien. State University of New York Press, USA, 1999. 274 pages]. Surfaces, 9.

https://doi.org/10.7202/1065071ar
Résumé de l'article

L'ouvrage part d'une prémisse simple : la culture fournit des outils pour vivre à la société. La logique soutenant le discours qui suit se construit comme une réponse qui se décline suivant une petite histoire du genre de l'horreur, comme le titre en illustre la portée : de Castle of Otranto jusqu'à Aliens. L'auteure est affiliée de recherche en études culturelles (cultural studies) à l'University of Manitoba. 


\section{Valdine Clemens - The Return of the Repressed: Gothic Horror from The Castle of Otranto to Alien}

Éric Alloi

Département de Littérature comparée Université de Montréal

Surfaces vol IX 1.03 (v.1.0F - 15/12/2001) - ISSN: 1188-2492

Tout texte reste la propriété de son auteur. Néanmoins, SURFACES demande d'être citée à l'occasion de toute autre publication du texte en question.

\section{RÉSUMÉ}

L'ouvrage part d'une prémisse simple: la culture fournit des outils pour vivre à la société. La logique soutenant le discours qui suit se construit comme une réponse qui se décline suivant une petite histoire du genre de l'horreur, comme le titre en illustre la portée: de Castle of Otranto jusqu'à Aliens. L'auteure est affiliée de recherche en études culturelles(cultural studies) à l'University of Manitoba.

\section{Valdine Clemens, The Return of the Repressed: Gothic Horror from The Castle of Otranto to Alien. State University of New York Press, USA, 1999. 274 pages.}




\section{INTRODUCTION}

Depuis que l'intérêt de la critique se tourne vers les foisonnantes paralittératures, nous découvrons l'importance de genres dont les enjeux culturels ont été trop longtemps ignorés. L'ouvrage de Clemens propose une réflexion sur cette problématique: "quel sont les rôles culturels et sociaux du genre littéraire de l'horreur?" L'ouvrage peut être considéré en deux parties. La première, surtout dans l'introduction, est une réflexion sur les rôles eux-mêmes et une considération des bases critiques utiles à leur analyse pour ce genre en particulier. La seconde est une illustration de la thèse présentée à l'aide d'une suite chronologique d'études de cas.

\section{LA THÈSE}

This study is based on the premise that literature provides what Kenneth Burque calls "equipment for living"...

La thèse de Clemens repose en grande partie sur l'utilisation de la psychanalyse pour comprendre le rôle social du récit d'horreur. Pour l'auteure, ce type de récit a comme fonction la plus évidente de servir de thérapie psychosociale. Son efficacité dépend de l'impact sur les affects du public. Se basant sur les théories de Jung, le public vit une régression qui permet de reprendre contact avec la psychée intérieure qu'une trop grande intellectualisation réprime. De là vient le titre The Return of the Repressed. L'atavisme est par conséquent le principal caractère de la consommation de littérature gothique d'horreur.

L'auteure traite aussi de la distinction entre le récit réaliste et le récit gothique. On attribue souvent la qualité d'analyse du social et du vécu quotidien aux romans réalistes. Cette caractéristique n'est généralement pas attribuée au récit gothique à cause de ses références constantes au passé. Par contre, il est possible de faire des liens très concluants entre les problématiques relevées dans le récit d'horreur et les enjeux sociaux de chaque époque. Nous pouvons dans ce cas parler de critique culturelle de la part des auteurs et l'intérêt de leurs oeuvres prend la forme d'un questionnement sur les tensions sociales de chaque 
époque telles que nous pouvons les relever sous couvert des situations effrayantes relatées dans chacunes.

Plus avant, la thèse de Clemens pose les divers types de situations, les courants et modes du genre comme marqueurs de changements sociaux importants(cultural shifts). Le sentiment intériorisé de transgression exprime les tensions vécues par ceux qui confrontent les codes sociaux des générations passées à celles en puissance. La peur ressentie vient entre autres de l'hésitation primaire entre le combat ou la fuite face à la nouveauté; le cauchemar est une auto évaluation d'un désir inadmissible en face des codes sociaux dont les institutions sociales en représentent l'autorité.

Parce que la fiction gothique d'horreur s'adresse à des problématiques contemporaines et qu'elle s'exprime à travers plusieurs médias, Clemens tient à prévenir le lecteur contre les dangers de la compartimentalisation des études sur cette partie de l'expression culturelle. Selon elle, une approche davantage multidisciplinaire serait souhaitable.

\section{LES OEUVRES ET L'HISTOIRE}

La culture occidentale montre des précédents de la "peur gothique" dès le Moyen-Âge. Mais le genre en tant que tel naît dans les années 1790 alors que la pensée des Lumières se trouve nostalgique de l'accès quotidien au surnaturel qu'elle attribue à l'existence médiévale. Dès lors, le mal dépeint dans les oeuvres littéraires est allégorique des forces qui menacent l'intégrité des institutions dont l'autorité s'affaiblit. Le château tient lieu de défense et son envahissement par les spectres témoigne de sa perméabilité au changement social.

Le Château d'Otrante est la première oeuvre de laquelle Clemens fait une analyse exhaustive. Le roman est publié en 1764 à une époque où le questionnement social porte sur l'institution de la famille. L'intrigue consiste en un chasser-croisé entre deux amants, culminant en un meurtre commis par erreur. Les tribulations du héros se terminent quand il abandonne le trône usurpé par ses ancêtres et que les deux amants de retirent chacun dans des couvents. À cette époque, le Parlement débat des lois sur les mariages dits clandestins. Le surnaturel, dans ce cas, reflète les problèmes familiaux et d'abus sexuels à 
connotations incestueuses qui font la plaie du dixhuitième siècle.

Le troisième chapitre commence par l'oeuvre d'Anne Radcliffe, A Sicilian Romance. Clemens ne se limite pas à ce récit en particulier, mais considère plutôt un ensemble d'oeuvres qui sont autant d'exemples de témoignages culturels. Les romans de la fin du dixhuitième siècle ont comme principal thème la fuite de femmes qui se sauvent de châteaux et monastères. La portée sociale de ce récit semble indiquer que les femmes ne sont plus satisfaites du rôle qu'on leur attribue. Leur dépendance économique et les abus de la part des hommes est relatée sous d'autres formes dans une littérature d'horreur gothique produite et consommée par les femmes en nombre sans précédent. La grandissante alphabétisation des femmes de classe moyenne peut expliquer la force de ce mouvement culturel. C'est à ce moment que Clemens précise la fonction psychosociale du récit gothique féminin de cette époque:

The reader's lack of interest in particularized psychological exploration suggests their implicit acceptance of the role gothic caracters tend to play as embodiments of social and psychic forces rather than as individual personnalities. (p.49)

Le chapitre suivant est consacré en entier à l'analyse de l'oeuvre de Matthew Lewis: The Monk. Cette fois, il s'agit d'une problématique d'une perspective masculine qui porte sur l'auto-répression et la censure publique. Fait intéressant, l'auteur commence par cacher discrètement son identité à la première édition. Il la révèle ensuite lorsque le roman se vend assez pour justifier une seconde édition et qu'il constate qu'il n'a pas particulièrement causé d'objections morales. Mais c'est alors qu'il se révèle en tant qu'auteur que les problèmes commencent. Il évite de justesse d'être accusé de blasphème, ce qui l'aurait possiblement conduit à une peine d'emprisonnement et à subir des châtiments corporels. Le malaise face à l'oeuvre vient du statut de législateur de Lewis. La critique se partage entre la défense et la reproche. Le roman ne peut faire autrement que de causer des remous chez les intellectuels anglais puisqu'il fait une critique implicite du caractère répressif de la politique contemporaine. Selon Clemens, le récit gothique fait partie d'un rituel social du changement de paradigme social: "Gothic fictions belong to the destructive phase of re-creation" (p.85). 
Le cinquième chapitre permet à l'auteure d'illustrer sa thèse à l'aide d'un des exemple les plus évidents. Il s'agit du Frankenstein de Mary Shelley. Le début du dixneuvième siècle vit une indistrialisation qui remodèle dramatiquement l'espace urbain. L'humain moderne impose sa volonté sur les éléments et voit son existence dénaturé. Le cauchemar, c'est la crainte d'une perte de contrôle luciférine ou icarienne, où la nature prendrait sa revanche. Le personnage de Frankenstein représente les angoisses émanant de la révolution industrielle. C'est la révolution démonisée. C'est aussi une réaction contre une scientifisation du vécu humain où le spirituel, négligé, ressurgit d'une manière dangereuse. Se tournant de nouveau vers Jung, Clemens nous fait remarquer que la personnalité double du docteur est de nature compensatoire. L'incendie de la maison de campagne du récit de Shelley peut être mis en parallèle avec l'exode rural résultant de l'industrialisation.

Le chapitre suivant, à l'aide du roman The Strange Case of Dr Jekyll and Mr Hyde de Stevenson, souligne un autre problème social dont le récit gothique d'horreur fait une problématique. Ici, les événements sont une réflexion sur la pratique de la prostitution féminine qui, à la fin du dix-neuvième siècle, commence à poser problème. L'autre thème abordé est celui de la mobilité sociale. Le personnage principal du roman est double. D'un côté philanthrope notoire et de l'autre, un pécheur caché. Le caractère effrayant de l'intrigue tient à ce que les deux personnages sont en réalité le même individu. Le parallèle avec la société vient du désaccord grandissant des valeurs face à la prostitution tolérée, pratiquée par les gens d'honnête réputation qui se partagent entre leur femme et la prostituée. Le complexe de "la madonne et la pute", jusqu'ici intégrée dans les moeurs, voit sa légitimité s'effriter. Le scandale de cette pratique se traduit, dans l'oeuvre de Stevenson, par l'horreur de l'avocat qui réalise comme le lecteur que Jekyll et Hyde sont la même personne.

Avec l'analyse suivante, portant sur Dracula, Clemens apporte un nouveau souffle critique en touchant la forme. En effet, Dracula peut être considéré comme un "media pastiche" dont les composantes sont les suivantes (p.154):

- découpures de journeaux contemporains

- transcription d'un journal intime

- lettres entre fiancés

- journal de bord d'un navire 
Le reste du chapitre est consacrée à la description du caractère reptilien de Dracula et à la fâcheuse tendance des personnages d'ignorer le caractère suspect des incidents. C'est d'ailleurs ce qui les mène à leur perte. Le récit peut se voir comme une critique de la rigidité de la pensée religieuse contemporaine. C'est aussi une critique de la pensée scientifique qui se donne comme explication autoritaire, mais souvent incorrecte. Le discours psychosocial de l'oeuvre peint une société qui rejette toute métaphysique au profit des dogmes scientifiques et religieux. Clemens rappelle que l'oeuvre de Stoker, presque prophétique, met en scène l'Angleterre envahie par un vampire, précédent de peu les événements de la Première Guerre Mondiale.

Alors que l'ouvrage est, jusqu'à ce point, consacré presqu'exclusivement à des oeuvres anglaises, les deux derniers chapitres se tournent vers le gothique américain. Stephen King, avec Shining (1977), porte aussi un regard critique sur la société. Clemens souligne les événements du scandale Watergate où le président Nixon avait joué un rôle important et la guerre du Viêtnam, deux crises dont l'horreur est bien réelle. Dans ces conditions, King décrit les événements qui se produisent dans une auberge dont une famille a la charge d'entretenir pendant les mois de saison morte. Les lieux sont hantés par des forces inexpliquées et d'une terrible violence. Clemens décrit la puissance d'une telle oeuvre dans ces termes (p. 186):

Because the nightmares images of Gothic fiction are in a sense the harbingers of a new awareness, it is caracteristic of reader response that initially people will react very strongly to a Gothic novel without fully understanding why they do so. This gap between reaction and comprehension is one of the reasons why The Shining, when it first appeared, was generaly regarded as a light but highly effective form of popular entertainment( another reason being the academic tendency to dismiss popular fiction as critically insignificant).

Clemens termine son ouvrage avec un épilogue portant cette fois toujours sur le gothique d'horreur, mais au lieu d'être surtout à caractère fantastique, il est maintenant question de science-fiction. En ce faisant, l'auteure fait le pont entre deux genres font le point commun gothique ouvre la thèse psychosociale sur un autre genre de la paralittérature. Alors que les précédentes oeuvres analysées sont tournées vers le passé, le genre du 
science fiction place son récit dans l'avenir. Clemens place d'ailleurs ce dernier genre dans la lignée du gothique d'horreur fantastique et insiste sur le fait que les fonctions d'avertissement et de critique sociale sont les mêmes. Le film Alien reprend la symbolique de la matrice et du noir, d'une esthétique chtonienne et menaçante, pour faire une critique du capitalisme libéral de la société de consommation motivé par l'appât du gain représentée par l'attitude de la compagnie qui engage les personnages du film.

\section{CONCLUSION}

Avec The Return of the Repressed, Celemens développe la thèse d'une fonction psychosociale du genre gothique d'horreur. Elle montre aussi l'importance de genres littéraires et artistiques souvent relégués au second plan par la critique. L'historique du genre, bien que centré sur les productions anglaises, montre bien la naissance d'une véritable tradition littéraire. Le lecteur qui ne souscrit pas complètement aux théories de Jung peut néanmoins, à la lecture de cet ouvrage concis, profiter d'une introduction remarquable au genre gothique. Les arguments soulevées par sa problématique s'inscrivent dans une perspective qui ne se limite pas à un champ critique en particulier sauront trouver des applications dans nombre de questionnements sur les rapports entre les oeuvres et l'évolution sociale.

Accueil Surfaces | Table des matières | Recherche Surfaces Home Page | Table of Contents | Search

PUM | Livres | Revues | Publications électroniques | Vente et distribution 